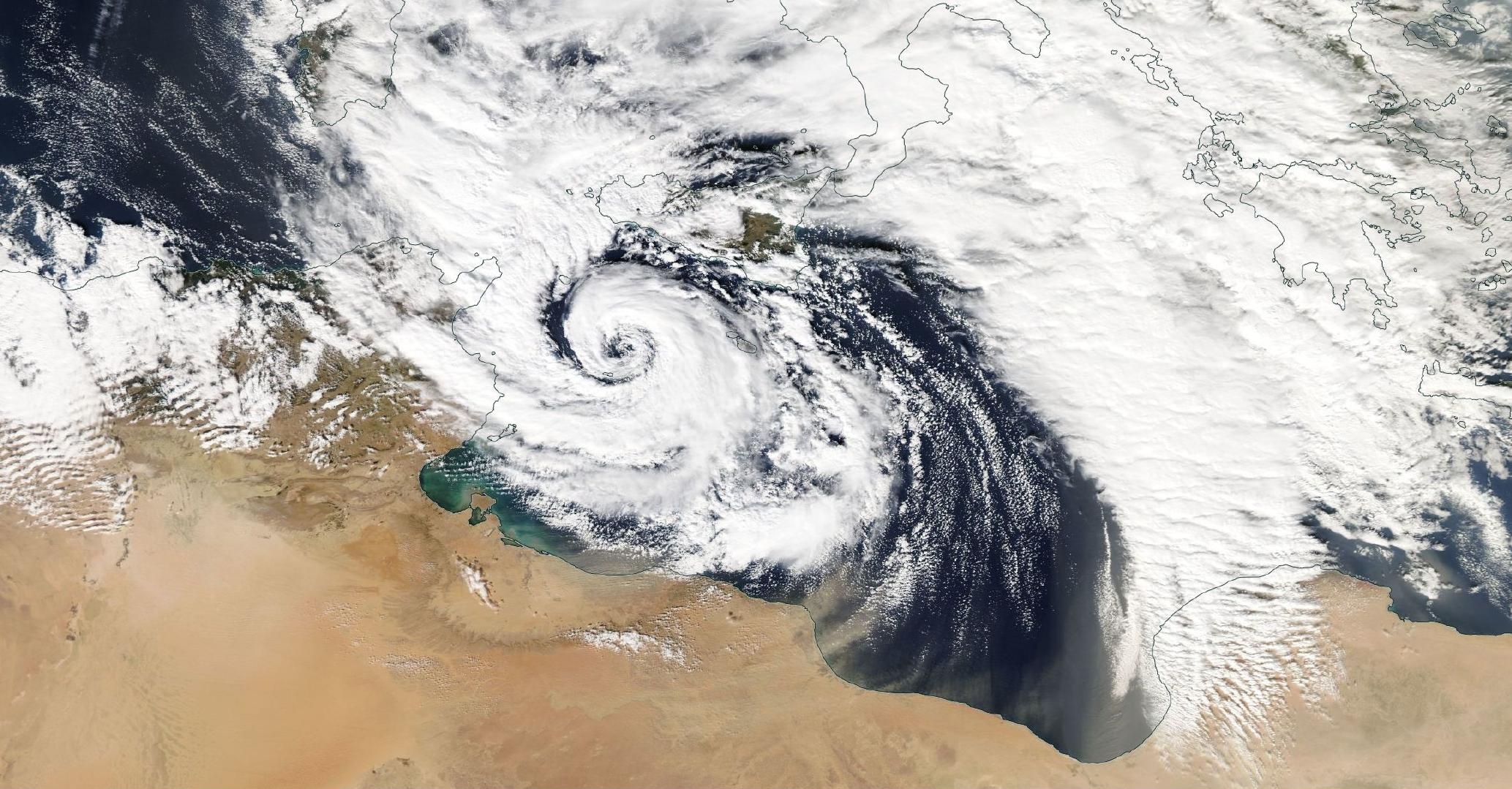

\title{
Predicción de medicanes
}

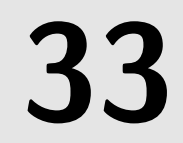

DOI: $10.31978 / 014-18-009-X .33$

Maria Angeles Picornell Alou y Joan Campins Pons

D. T. en las islas Baleares, Agencia Estatal de Meteorología (AEMET)

Mon cor estima un arbre! Més vell que l'olivera, més poderós que el roure, més verd que el taronger, conserva de ses fulles l'eterna primavera, i lluita amb les ventades que atupen la ribera, com un gegant guerrer.
Oh! sí: que quan a lloure bramulen les ventades i sembla entre l'escuma que tombi el seu penyal,

... llavors ell riu i canta més fort que les onades, i vencedor espolsa damunt les nuvolades sa cabellera reial.

El pi de Formentor - MIQuel Costa I LloberA

Los sistemas de previsión meteorológica actuales encuentran su gran caballo de batalla en los fenómenos extremos, aquellos que ocurren con relativa poca frecuencia y los fenómenos adversos, aquellos que producen mayor impacto social y económico. Los fenómenos extremos no siempre son adversos y, viceversa, no todo fenómeno adverso es raro, pero tienen algo en común: la complicación e importancia especiales a la hora de predecirlos con el acierto y la antelación necesarios. Los sistemas de predicción por conjuntos (SPC) pueden ayudar a prever estos fenómenos enfocando la predicción de forma probabilista, especialmente si se desarrollan herramientas específicas basadas en ellos. En este capítulo presentamos una metodología desarrollada específicamente en AEMET para la previsión de un fenómeno adverso típicamente mediterráneo: los mediterranean hurricanes o medicanes.

Palabras clave: sistemas de predicción por conjuntos aplicados en previsión de medicanes, mediterranean hurricanes o medicanes, ciclones mediterráneos y tiempo adverso, detección y trayectoria de ciclones, espacio de fases de ciclones.

Imagen parte superior: imagen True-Color del sensor MODIS del satélite Aqua (NASA) del 7 de noviembre de 2014, aproximadamente a las 12 UTC, con un medicane entre Túnez y Sicilia. Imagen extraída de la aplicación de la NASA Worldview (https://worldview.earthdata.nasa.gov/), NASA/Goddard Space Flight Center Earth Science Data and Information System (ESDIS) project. 


\subsection{Los ciclones mediterráneos y el tiempo adverso}

Desde el punto de vista meteorológico, el Mediterráneo es una región peculiar, diferente a otras regiones del mundo. Esto se debe principalmente a factores morfológicos y geográficos. En primer lugar se trata de un mar casi cerrado y relativamente cálido, rodeado de una compleja orografía, en la que se alternan elevadas cordilleras y estrechos valles, y a la presencia de golfos, islas y penínsulas de muy diversos tamaños. Todo ello tiene importantes consecuencias sobre los fenómenos atmosféricos porque propicia una gran variabilidad espacial y temporal, resaltando sobre todo las características mesoescalares (sec. 4.3.2 en la página 39). En segundo lugar, su posición geográfica coloca a la región bajo la influencia de diferentes circulaciones de gran escala, como las asociadas a las propias de latitudes medias o las circulaciones tropicales o subtropicales. Estas circulaciones de gran escala pueden verse modificadas por la morfología de la región y a su vez también pueden interactuar con las propias de menor escala espacial [12].

Entre las peculiaridades de la región mediterránea se encuentra su elevada capacidad de generar ciclones o de rejuvenecer a los ciclones que provienen de otras regiones, especialmente del Atlántico Norte, pero también del norte de África ([12] y cap. 32 en la página 531). Aunque el término ciclón en general se suele asociar a los ciclones tropicales, se utiliza de un modo más amplio como sinónimo de depresión, borrasca (si es grande e intenso), centro ciclónico, centro de baja presión o simplemente baja. En el Mediterráneo existen zonas preferentes de formación de ciclones (como el golfo de Génova o Chipre), así como trayectorias preferentes de movimiento de dichos ciclones [2]. Por lo que respecta al tamaño y la duración, los ciclones mediterráneos suelen ser menores que los ciclones atlánticos (predominando los de mesoescala o escala subsinóptica frente a la sinóptica) y de vida más corta (del orden de un día frente a varios días). Muchos de estos ciclones son poco intensos y de poco espesor vertical, como los ciclones a sotavento de las cordilleras o las bajas térmicas. Otros, menos frecuentes, son intensos y profundos y en muchos casos se trata de ciclones extratropicales típicos que se forman en la propia región o provienen de regiones próximas. Estos ciclones son de tipo frontal (aunque los frentes en el Mediterráneo son, a menudo, difíciles de identificar) y su mecanismo de formación o desarrollo es la inestabilidad baroclina. Finalmente cabe resaltar otro tipo de ciclón, en este caso pequeño pero muy intenso, que comparte algunas similitudes con los ciclones tropicales y que por este motivo se denominan ciclones casi-tropicales o medicanes (de medi-terranean hurri-cane [5]). Estos ciclones se forman sobre el mar y para su desarrollo adquieren la energía a partir de los flujos superficiales de calor sensible y latente y de la liberación de calor latente de las nubes convectivas. Por último, es importante señalar que los diversos mecanismos ciclogenéticos (orografía, inestabilidad baroclina, liberación de calor latente, etc.) pueden aparecer simultáneamente o en diferentes fases del desarrollo de un mismo ciclón mediterráneo [12].

En el Mediterráneo es conocida la relación que existe entre episodios de tiempo adverso y los ciclones y en muchas ocasiones estos episodios tienen un alto impacto social y económico [11]. En muchos casos se trata de ciclones relativamente grandes e intensos como por ejemplo el que afectó a Argelia y a la costa este española, sobre todo a las islas Baleares, entre el 9 y el 11 de noviembre de 2001. Este ciclón produjo intensas precipitaciones y vientos muy fuertes tanto en tierra como en el mar. En Argelia las inundaciones asociadas provocaron la muerte de centenares de personas y cuantiosas pérdidas materiales. En las Baleares también se produjeron importantes precipitaciones, pero los principales efectos fueron el fortísimo viento que causó graves desperfectos en zonas costeras [7] y se estima que en Mallorca arrancó un millón de árboles. Otro ejemplo, más próximo en el tiempo, lo encontramos en el temporal de lluvia, nieve, viento y olas que afectó principalmente a la Comunidad Valenciana y a Baleares en enero de 2017 (cap. 52 en la página 795). En otros casos, mucho menos frecuentes, el tiempo adverso está producido por pequeños e intensos ciclones, como por ejemplo los medicanes [18]. Más adelante trataremos de forma extensa un ejemplo de este tipo de ciclones (subsección 33.4 en la página 556).

Pero no solo los ciclones intensos mediterráneos están relacionados con el tiempo adverso. En ocasiones ciclones pequeños y poco intensos juegan un papel clave en episodios de lluvias intensas. Estos ciclones pueden canalizar o focalizar un flujo de aire cálido y húmedo que sirva como alimentación de las precipitaciones. Este sería el caso, por ejemplo, del récord de precipitación registrado en Oliva (Valencia) entre el 3 y 4 de noviembre de 1987 (cap. 44 en la página 655). Este marco conceptual se ha comprobado válido en muchos casos de lluvias fuertes en el Mediterráneo occidental, sobre todo en las regiones mediterráneas 
españolas [10].

\subsection{Predecibilidad de los ciclones mediterráneos}

La predicción numérica está mejorando continuamente: aumenta la resolución de los modelos, se utiliza mayor número de observaciones (con lo que se mejoran las condiciones iniciales) y también progresan los métodos de asimilación y predicción de los mismos. Aún así, sabemos que en los episodios de baja predecibilidad el error de predicción de los modelos deterministas puede ser elevado. Por este motivo el uso de modelos probabilistas nos puede ayudar a mejorar la predicción y a cuantificar la incertidumbre que esta conlleva. Todo ello puede ser especialmente útil en los casos de tiempo adverso (caps. 12 en la página 155 y 13 en la página 165 ).

Una de las principales fuentes de error de los modelos numéricos es una insuficiente caracterización del estado inicial de la atmósfera (cap. 16 en la página 243). Este tipo de error se ve acentuado en el Mediterráneo debido a que una parte importante de esta región se sitúa sobre el mar y a que además amplias regiones cercanas a la misma también carecen de una buena cobertura de observaciones (por ejemplo el Atlántico Norte o el norte de África). Otro aspecto a tener en cuenta es cómo crecen estos errores. Así por ejemplo, en latitudes medias, en predicciones a medio plazo (entre 3-5 días) y a escala sinóptica el crecimiento de los errores está asociado (entre otras causas, aunque principalmente) a la inestabilidad baroclina y estos errores crecen sobre todo en los primeros días de predicción. De este modo las incertidumbres en las condiciones iniciales pueden situarse lejos de la región de interés, o en el caso de los modelos de área limitada incluso fuera de su dominio (en este caso las incertidumbres "entrarían" a través de las condiciones de contorno). Los errores asociados a la inestabilidad baroclina se ven incrementados por procesos en los que interviene la humedad. Los tipos y ritmos de crecimiento de los errores en las predicciones a corto plazo y para escalas menores son diferentes a los de escalas mayores, aunque de nuevo los procesos dinámicos relacionados con la humedad tienen un papel muy destacado (cap. 17 en la página 257).

En los últimos años se han realizado diversos estudios sobre el error y la predecibilidad de los ciclones extratropicales. Quizás el más importante es el que se desarrolló en el marco del proyecto TIGGE (cap. 26 en la página 387) en el que se compararon 9 ensembles globales (se puede consultar un resumen de los resultados en FROUDE, 2010 [6]). En el mismo se demuestra que el ECENS es el mejor SPC global a la hora de predecir la posición, la intensidad y la velocidad de propagación de los ciclones, aunque con importantes limitaciones. El ECENS muestra un mayor acierto a la hora de predecir la posición que la intensidad de los ciclones extratropicales. Además, el error en la posición es debido principalmente la velocidad de propagación (los ciclones previstos son más lentos que los analizados) y no tanto a la dirección. Como ocurre con la mayoría de ensembles, tanto el error como la predecibilidad de los ciclones extratropicales aumenta con el tiempo y en general la relación dispersión-error (sec. 15.8.2 en la página 223) muestra ser buena para la predicción de la trayectoria (el error aumenta al mismo ritmo que la dispersión) pero no para la intensidad, donde el error crece más rápido que la desviación estándar del SPC (es decir el modelo es subdispersivo).

Estas conclusiones son generales para latitudes medias y, por tanto, también son aplicables a los ciclones mediterráneos, aunque conviene señalar que estos estudios se centraron en los ciclones grandes (sinópticos o subsinópticos), móviles y de duración de varios días, es decir, se centraron en una fracción de los ciclones mediterráneos, aunque probablemente asociados a tiempo adverso. Por otro lado hay que tener en cuenta que estos estudios se realizaron con predicciones de hace unos años (antes del 2010) por lo que es muy probable que gracias a las mejoras realizadas en el ECENS, actualmente sus predicciones también hayan mejorado. Desgraciadamente en el ECMWF no existe todavía una verificación de la predicción de los ciclones extratropicales, pero sí la hay para los ciclones tropicales, tanto para el ECHRES como para el ECENS. Para los ciclones tropicales se comprueba una mejora a lo largo del tiempo en la predicción de su trayectoria y de su intensidad (mayor en la trayectoria) y para ambos modelos, sobre todo a partir del 2010 [8].

Hasta el momento nos hemos referido a la predicción probabilista realizada a partir de ensembles globales para predicciones a medio plazo de ciclones extratropicales. Nos podemos preguntar ahora cómo predicen los SPC, tanto globales como de área limitada y de medio o de corto plazo, los ciclones mediterráneos, sobre todo los más pequeños e intensos. Por lo que conocemos, no existe un estudio sistemático sobre los 
errores en la predicción de los ciclones mediterráneos o sobre su predecibilidad. Un primer intento de estimar la habilidad para predecir ciclones mediterráneos a partir de modelos deterministas (primero para el año 1999, a partir del HIRLAM-INM de $50 \mathrm{~km}$, y después para el 2006-2007, con ECHRES de 25 km de resolución) se realizó utilizando las herramientas de detección y seguimiento que se explican a continuación [16]. En estos trabajos se obtienen diferentes medidas (scores) de verificación (sec. 15.2.3 en la página 211) de los ciclones previstos respecto a los analizados y se evalúa el error para diversos rangos de predicción. Por otro lado sí existen muchos estudios de episodios concretos que exploran los diferentes aspectos que provocan mayores errores o aumentan mucho la incertidumbre de los ciclones mediterráneos. El capítulo 32 en la página 531, que trata sobre los SPC y el Mediterráneo, contiene algunas reflexiones sobre la predecibilidad de los ciclones mediterráneos en general y los medicanes en particular.

\subsection{Predicción de ciclones a partir de modelos numéricos}

\subsubsection{Detección y trayectoria de ciclones}

Durante las últimas décadas, el aumento de la resolución y la calidad de los modelos numéricos de predicción meteorológica ha posibilitado la descripción cada vez más precisa de estructuras atmosféricas de tamaño cada vez menor. Por otra parte, la ya mencionada relación de algunos centros ciclónicos con el tiempo atmosférico, y en particular su influencia en la localización e intensidad de ciertos fenómenos que pueden ser adversos, ha alimentado el interés en representar estas estructuras. Todo ello ha alentado el desarrollo de diversos procedimientos para identificar estructuras ciclónicas y seguir su trayectoria, de manera automática y objetiva, a partir de los campos meteorológicos proporcionados por los modelos numéricos. En estos procedimientos, desarrollados para diversas regiones geográficas, se han utilizado diferentes criterios para definir un ciclón y para determinar su trayectoria [13].

En Agencia Estatal de Meteorología (AEMET) se desarrolló un procedimiento de detección y seguimiento de ciclones que afectan a la región mediterránea $[1,17]$. En este método se identifica como ciclón un mínimo del campo de presión a nivel del mar con un cierto gradiente a su alrededor. Una vez identificado y, para seguir su trayectoria, se asume la hipótesis de que se desplaza dirigido por el viento a niveles medios. Se imponen unas condiciones de selección poco restrictivas, con el fin de detectar tanto ciclones mesoescalares intensos como pequeñas estructuras o ciclones poco intensos y poder analizar su influencia en el tiempo atmosférico. Esto provoca que un gran número de ciclones sean identificados en el Mediterráneo, algunos de ellos sin consecuencias relevantes. Estos criterios pueden modificarse para describir determinados tipos de ciclones, así como completarse con otros parámetros para describir su estructura tridimensional. De hecho a lo largo de estos años el procedimiento ha ido sufriendo una serie de modificaciones para adaptarlo a los diferentes modelos numéricos utilizados. Se han obtenido climatologías a partir del reanálisis ERA40 [19], se ha utilizado para obtener ciclones previstos a partir de modelos de predicción, principalmente deterministas, como el HIRLAM, con diferentes resoluciones, y el ECHRES, y a partir del modelo probabilista AEMET-SREPS en modo experimental.

\section{Criterios para identificar un ciclón}

- Se define como ciclón un mínimo de presión a nivel del mar, con $\Delta p \geq 0,5 \mathrm{hPa} / 100 \mathrm{~km}$ al menos en seis de las 8 direcciones principales alrededor del mínimo. Para eliminar el ruido excesivo que presentan algunos campos derivados, como el de la vorticidad, se suaviza el campo de presión mediante un filtro de CRESSMAN [4], con un radio adecuado a la resolución del modelo utilizado.

- Definimos el dominio del ciclón como la región limitada por la línea de vorticidad geostrófica nula que lo rodea, y calculamos su radio medio y su circulación geostrófica. Si existe un núcleo cálido en la vertical del ciclón en la troposfera media, se calcula también su radio medio a partir de la línea de laplaciana nula de temperatura.

- Para determinar la trayectoria de los centros ciclónicos se utiliza un área de búsqueda elíptica orientada según el viento en $700 \mathrm{hPa}$.

\subsubsection{El espacio de fases de los ciclones}

Ante la diversidad de tipos de estructuras ciclónicas observados, cada uno de ellos con sus características propias, HART presenta en 2003 [9] una nueva herramienta que permite clasificar los ciclones a partir de su estructura térmica tridimensional, válida para todo el espectro de ciclones, desde ciclones puramente tropicales a ciclones extratropicales baroclinos. 
Propone el denominado espacio de fases de los ciclones, EFC, (cyclone phase space, CPS, en inglés), en el que la posición que ocupa un ciclón, la fase del ciclón, informa de su tipología y su ciclo de vida puede ser analizado dentro de este espacio, proporcionando información sustancial sobre la evolución de su estructura.

Este espacio se construye a partir de tres parámetros: la asimetría térmica a niveles bajos (parámetro B), el viento térmico de nivel bajo (parámetro $-V_{T}^{L}$ ) y el viento térmico de la troposfera alta $\left(-V_{T}^{U}\right)$.

El parámetro B informa de la naturaleza frontal del ciclón, propia de ciclones baroclinos, con fuerte gradiente de temperatura $(B>10 \mathrm{~m})$, o de la ausencia de ella, como ocurre en los ciclones tropicales, térmicamente simétricos y con un valor de $\mathrm{B}$ próximo a cero.

Los otros dos parámetros, el viento térmico de nivel bajo (parámetro $-V_{T}^{L}$ ) y el viento térmico de la troposfera alta $\left(-V_{T}^{U}\right)$, permiten distinguir si la estructura vertical corresponde a un núcleo cálido profundo (warm-core), es decir a un ciclón de tipo tropical, o si existe un núcleo frío (cold-core), propio de un ciclón extratropical. Se trata de un esquema simplificado y, por tanto, es difícil que pueda abarcar todos los casos posibles.

Para facilitar la visualización, el EFC se presenta mediante dos secciones trasversales, los llamados diagramas de fase (DF). En uno de ellos se representa $B$ versus $-V_{T}^{L}$, y en el otro $-V_{T}^{U}$ versus $-V_{T}^{L}$. Se definen cuatro zonas en los DF, en las que se clasifican los diferentes tipos de ciclones, aunque no hay una separación estanca entre estas regiones.

El ciclo de vida completo del ciclón se define a partir de su trayectoria en los DF. Estos diagramas pueden incluir además información adicional referente a la profundidad y al radio del ciclón.

Si bien estos diagramas muestran características esenciales de los centros ciclónicos, para obtener una descripción completa de los mismos es necesaria información complementaria, como la suministrada por los productos de teledetección.

\section{Parámetros del Espacio de Fases de los Ciclones (EFC)}

HART [9] calcula estos parámetros descritos anteriormente en un círculo de $500 \mathrm{~km}$ de radio alrededor del centro ciclónico y recomienda utilizar un círculo variable que se ajuste al tamaño del ciclón.

Además, para ello examina dos capas troposféricas de igual masa: 900-600 hPa y 600-300 hPa. Para los ciclones mediterráneos, de menores dimensiones que los tropicales, utilizamos un círculo menor.

Si existe núcleo cálido a nivel medio, se utiliza su radio como radio del círculo de exploración. En caso contrario, utilizamos un círculo de $100 \mathrm{~km}$ de radio.

Consideramos también que en esta región las capas de exploración entre 925 y $700 \mathrm{hPa}$ y entre 700 y $400 \mathrm{hPa}$ se ajustan mejor al espesor de la tropopausa, menor en el Mediterráneo que en los trópicos [15].

- Parámetro B: para calcularlo se dividide el círculo por la bisectriz en la dirección de su desplazamiento. La magnitud de B se obtiene como la diferencia de espesor del estrato inferior entre el semicírculo a la derecha del movimiento y el de la izquierda. Este parámetro mide el gradiente de la temperatura media del estrato perpendicular al desplazamiento del ciclón.

- Viento térmico en la baja troposfera: $-V_{T}^{\mathrm{L}}$. Se calcula a partir del perfil vertical del gradiente de altura geopotencial en el estrato inferior.

- Viento térmico en la troposfera medio-alta: $-\mathbf{V}_{\mathbf{T}}^{\mathbf{U}}$. Esta medida ayuda a distinguir ciclones de núcleo cálido profundo (de tipo tropical) de los ciclones con núcleo cálido poco profundo (como las seclusiones cálidas o ciclones subtropicales).

Ciclones mediterráneos

$B={\overline{\left.\left(Z_{700}-Z_{925}\right)\right|_{L}}}-\left.\overline{\left(Z_{700}-Z_{925}\right)}\right|_{R}$
$\left.\frac{\partial(\Delta Z)}{\partial \ln p}\right|_{925 h P a} ^{700 h P a}=-\left|V_{T}^{L}\right|$
$\left.\frac{\partial(\Delta Z)}{\partial \ln p}\right|_{700 h P a} ^{400 h P a}=-\left|V_{T}^{U}\right|$




\subsection{Un caso particular: Medica- nes}

Actualmente la comunidad científica está trabajando para conseguir una definición consensuada de lo que es un medicane y de qué características son esenciales para identificarlos. Se ha observado que los medicanes son pequeños ciclones que se desarrollan sobre el mar y afectan principalmente a zonas marítimas, islas y zonas costeras. Pueden provocar vientos muy fuertes, temporales marítimos e incluso inundaciones, resultado de lluvias muy intensas. Se los identifica por una estructura nubosa muy característica, con forma de espiral ciclónica, casi circular, con intensa actividad convectiva y con una zona central sin nubes, el ojo del medicane, que recuerda a los ciclones tropicales pero con un tamaño mucho menor. Algunas de estas características pueden evaluarse mediante las herramientas que acabamos de describir. En este apartado proponemos una definición objetiva de un posible medicane, o mejor de un «candidato» a medicane, a partir de las salidas de un modelo numérico. Así pues, definimos un posible medicane como un ciclón muy intenso, con un fuerte gradiente de presión, que presenta una estructura cálida simétrica y profunda. Este candidato deberá cumplir además otras condiciones que deberán evaluarse a partir de otras fuentes, como pueden ser los productos de teledetección.

Definición de medicane a partir de los campos de un modelo numérico

- Ciclón muy intenso, $\Delta p \geq 3,2 \mathrm{hPa} / 100 \mathrm{~km}$ en al menos 6 de las 8 direcciones. Este gradiente corresponde aproximadamente a un viento geostrófico medio de unos $32 \mathrm{~m} / \mathrm{s}$.

- El ciclón debe tener una estructura térmica simétrica, es decir, $B<10 \mathrm{~m}$.

- El ciclón debe presentar una estructura de núcleo cálido profunda, $-V_{T}^{L}>0 \mathrm{y}-V_{T}^{U}>0$.
Si utilizamos las herramientas descritas hasta ahora con modelos numéricos de suficiente resolución, ya sean deterministas o probabilistas, podemos obtener una predicción objetiva y sistemática (basada en una descripción cuantificada, que permite una identificación objetiva de la tipología del ciclón) de los ciclones mediterráneos, y en particular de los medicanes. La predicción por conjuntos de los medicanes puede aportar una valiosa información sobre las posibles evoluciones que puede seguir el ciclón y de la probabilidad con la que podemos esperar cada una de ellas. Cada uno de los miembros del ensemble predice una evolución de ciclón, con lo cual tendremos tantas trayectorias posibles como miembros (por ejemplo como las que se muestran para el ECENS en la Figura 33.1 en la página siguiente). Esta información es especialmente útil al permitir aumentar la vigilancia en zonas que potencialmente pueden verse afectadas. Además, la gran cantidad de información suministrada a partir de los modelos probabilistas debe ser presentada de manera concisa y clara, resaltando las características predominantes pero sin dejar de mostrar los posibles valores extremos aunque tengan baja probabilidad. El mapa de la trayectoria prevista para un ciclón se convierte ahora en un mapa de densidad de trayectorias previstas, que nos informa de las zonas por las que puede pasar el ciclón con una cierta probabilidad. Las características del ciclón previsto, como su radio, el valor de la presión en el centro o su tiempo de vida, pasan a tener un rango de valores posibles y el espacio de fases presenta un abanico de evoluciones y transiciones previstas. Presentamos a continuación una serie de productos obtenidos para la predicción de un medicane que ha sido detectado y caracterizado a partir de los campos analizados y previstos del modelo ECHRES y por el ECENS. Se trata de productos similares a los desarrollados por el ECMWF para la predicción probabilista de ciclones tropicales ([14], sec. 27.7.4 en la página 422 y cap. 46 en la página 717). 

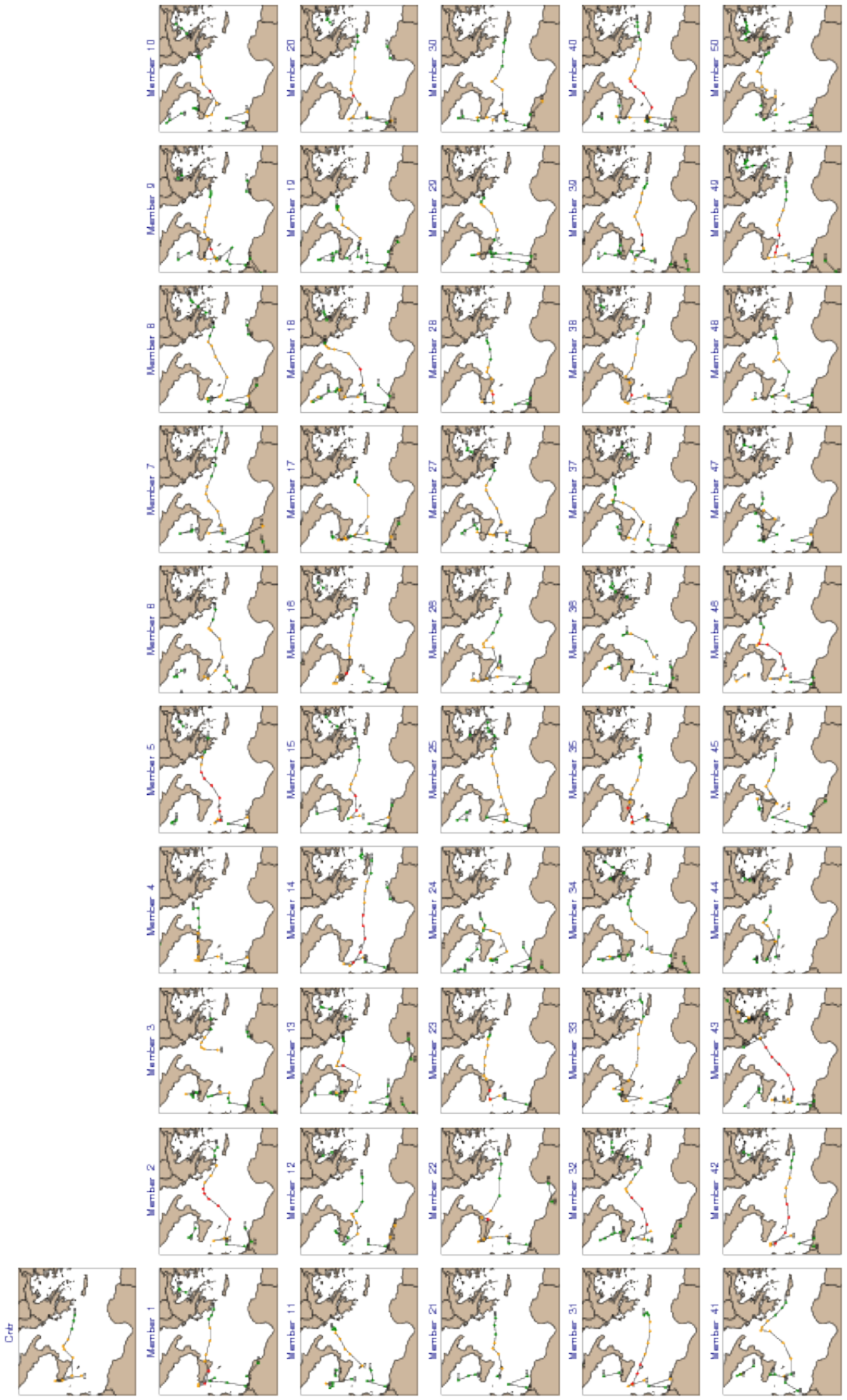

Figura 33.1: Sellos de la trayectoria de los ciclones previstos por cada miembro del ECENS. La intensidad del ciclón en cada posición determina el color del círculo: débiles-moderados $(\Delta p<1,5 \mathrm{hPa} / 100 \mathrm{~km})$ en verde, intensos $(1,5 \geq \Delta p<3,2 \mathrm{hPa} / 100 \mathrm{~km})$ en naranja y muy intensos $(\Delta p \geq 3,2 \mathrm{hPa} / 100 \mathrm{~km})$ en rojo. 

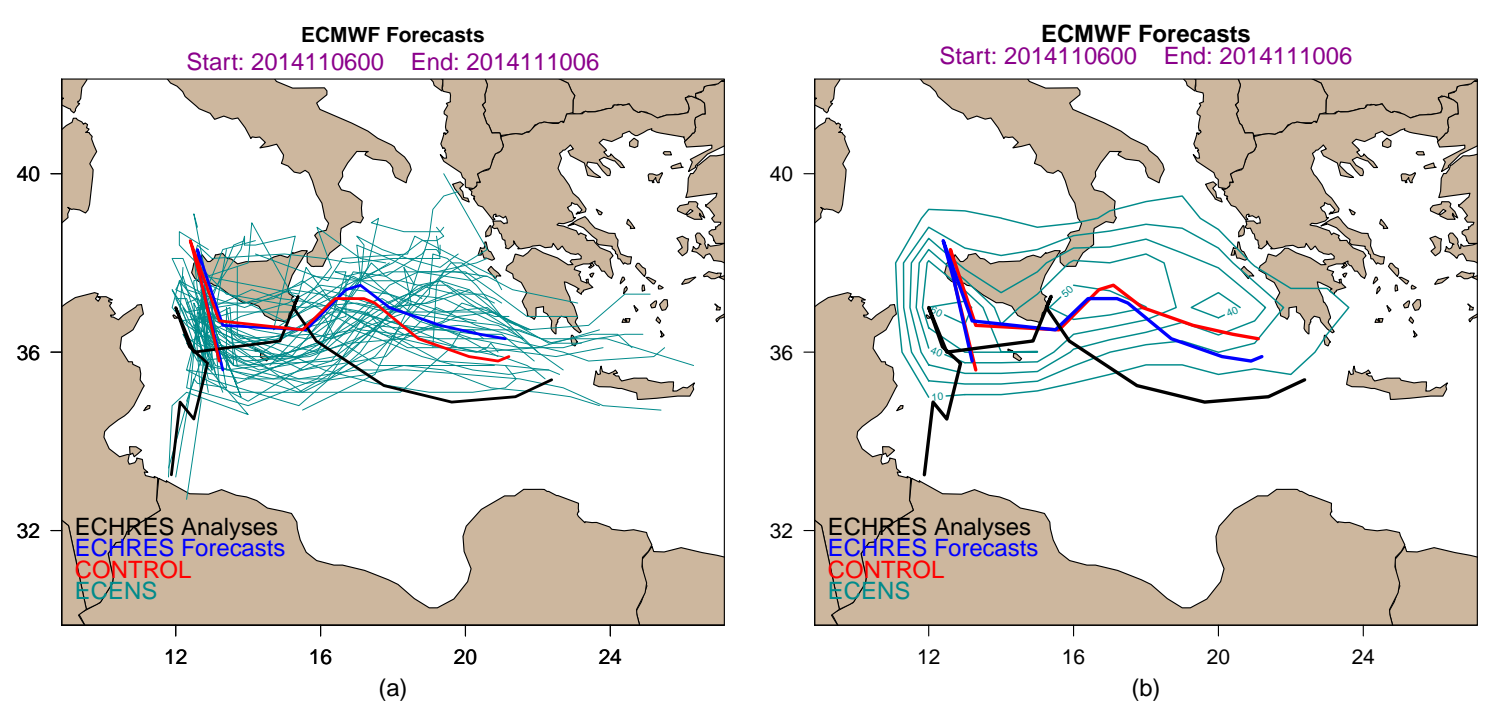

Figura 33.2: Izquierda: espaguetis correspondientes a las trayectorias previstas por los diferentes miembros del ECENS (32 km de resolución horizontal). Derecha: mapa de probabilidad de trayectorias. En ambas figuras se muestran las trayectorias previstas y analizadas por el ECHRES (16 km de resolución horizontal).

Se trata de un medicane, al menos en buena parte de su trayectoria, que afectó al Mediterráneo central los días 7 y 8 de noviembre de 2014, descrito en Carrió et al (2017 [3]). Su trayectoria y la evolución de la presión en el centro del ciclón se muestran en las Figuras 33.2 y 33.4 en la página siguiente. El ciclón se formó durante la madrugada del día 6 frente a la costa tunecina. Según el análisis del ECHRES, durante las primeras horas se desplazó hacia el norte y a partir de las 18 UTC profundizó rápidamente, con un caída de presión en su centro de $17.4 \mathrm{hPa}$ en 18 horas. El ciclón cambió bruscamente de dirección para desplazarse hacia el SE describiendo un pequeño bucle. El valor más bajo de presión se alcanzó día 7 a las 12 UTC y en las horas siguientes se registraron fuertes vientos y lluvias intensas en las islas que encontró a su paso. En Malta se registraron rachas de viento que superaron los $42,7 \mathrm{~m} / \mathrm{s}$, y una caída de presión de $20 \mathrm{hPa}$ en 6 horas, con un valor de presión de $985 \mathrm{hPa}$ a las 16:45 UTC. En su trayectoria rodeó la isla de Sicilia y afectó a su costa oriental. En ese momento el ciclón empezó a debilitarse y se desplazó hacia el este.

Su evolución en los DF (ver Figura 33.6 en la página 561) revela la presencia de un núcleo cálido simétrico y profundo desde el día 7 a las 12 UTC hasta el día 8 a las 00 UTC, es decir, durante esas horas el ciclón puede ser clasificado como un medicane de acuerdo con la definición propuesta.

Las previsiones del ECHRES para ese día muestran la presencia de un ciclón en la zona (ver Figura 33.2). Como ya se ha mencionado, cada uno de los miembros del ECENS predice una evolución del ciclón, con lo que nos presenta 51 escenarios posibles (ver Figura 33.1 en la página anterior). Durante las primeras horas, en las que el ciclón es poco intenso, tanto el ECHRES como muchos miembros del ensemble detectan centros ciclónicos en la zona pero no los relacionan con el ciclón que detectan por primera vez el día 6 a las 18 UTC. Agrupamos en un mismo mapa las trayectorias de aquellos ciclones previstos por los diferentes miembros que son intensos en algún momento de su evolución (ver Figura 33.2 izquierda) y resaltamos la trayectoria prevista por el miembro de control del ECENS. Esta figura nos informa de la dispersión que existe en las trayectorias previstas. A partir del mapa de densidad de trayectorias cuantificamos la probabilidad de que el ciclón se desplace por una zona determinada que se muestra en la Figura 33.2 derecha. En ambas figuras añadimos también la trayectoria del ciclón analizado y la trayectoria prevista por el ECHRES, con el fin de compararlas. ECHRES y control prevén una trayectoria del ciclón muy parecida, que es similar a la analizada pero con algunas diferencias: está desplazada unos grados hacia el norte respecto a la analizada y si bien reproduce los bucles que recorre el ciclón, a partir de día 8 a las 00 UTC las velocidades de desplazamiento son diferentes, el ciclón se desplaza más lentamente en los campos previstos que en el analizado, lo que se refleja en un desfase longitudinal. 


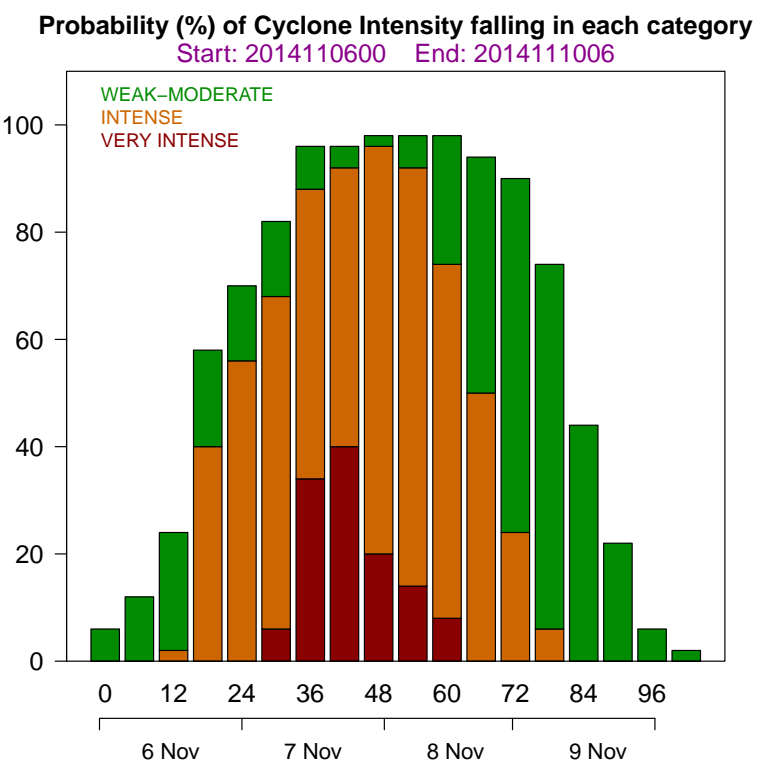

Figura 33.3: Probabilidad (\%) de que la intensidad del ciclón previsto por el ECENS sea débil o moderada, fuerte o muy fuerte.

Las trayectorias previstas se alejan de la analizada a medida que aumenta el rango de predicción. En este caso la zona de mayor dispersión coincide con una zona marítima, lo que plantea algunas dudas sobre si el carácter marítimo propio de los medicanes supone una dificultad añadida a su predicción.

Clasificamos los ciclones en tres grupos según su intensidad y obtenemos un histograma que nos informa del número de miembros que detectan ciclón en cada periodo de predicción (probabilidad) así como de su intensidad (ver Figura 33.3). Durante las primeras y las últimas horas del ciclo de vida del ciclón, momentos en los que el ciclón es más débil, pocos miembros lo detectan. Más de un $40 \%$ de los miembros del ensemble sí lo detectan entre $\mathrm{H}+18$ y $\mathrm{H}+84$, y más del $80 \%$ entre $\mathrm{H}+30$ y $\mathrm{H}+72$. Por otra parte, para los momentos de mayor intensidad del ciclón, entre las 12 UTC del día $7(\mathrm{H}+36)$ y las 00 UTC del día $8(\mathrm{H}+48)$, el SPC predice un ciclón que será como mínimo intenso con una probabilidad de casi el $100 \%$, y con una probabilidad en torno al $30 \%$ de que sea incluso muy intenso.

Los diferentes miembros del ensemble predicen un rango de valores posibles para las características del ciclón, como la presión en el centro, el máximo gradiente de presión o el viento térmico. En las Figuras 33.4 y 33.5 en la página siguiente se muestra la distribución de estos valores y de nuevo la comparamos con los valores analizados y con los previstos por el modelo de alta resolución.
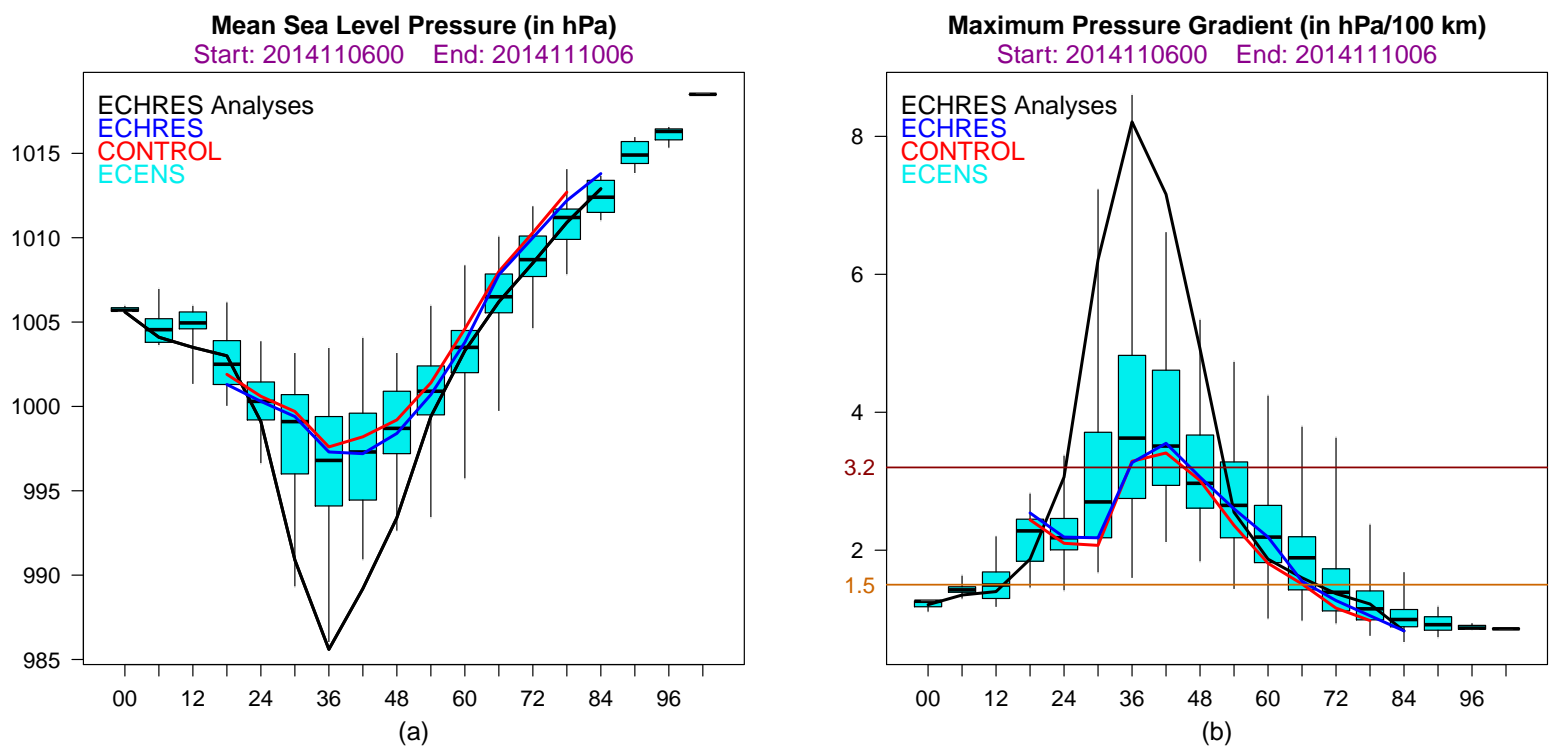

Figura 33.4: EPSgrama de la presión (izquierda) y el gradiente máximo de presión (derecha) para los 50 miembros perturbados del ECENS. El boxplot muestra una caja rectangular cuyos bordes superior e inferior son el percentil 75 y 25 respectivamente y una linea horizontal en su interior que muestra la mediana. Las líneas verticales superior e inferior tiene como extremo los valores máximo y mínimo respectivamente. Se muestra también la evolución de estas variables prevista por el miembro de control y las prevista y analizada por el ECHRES. 

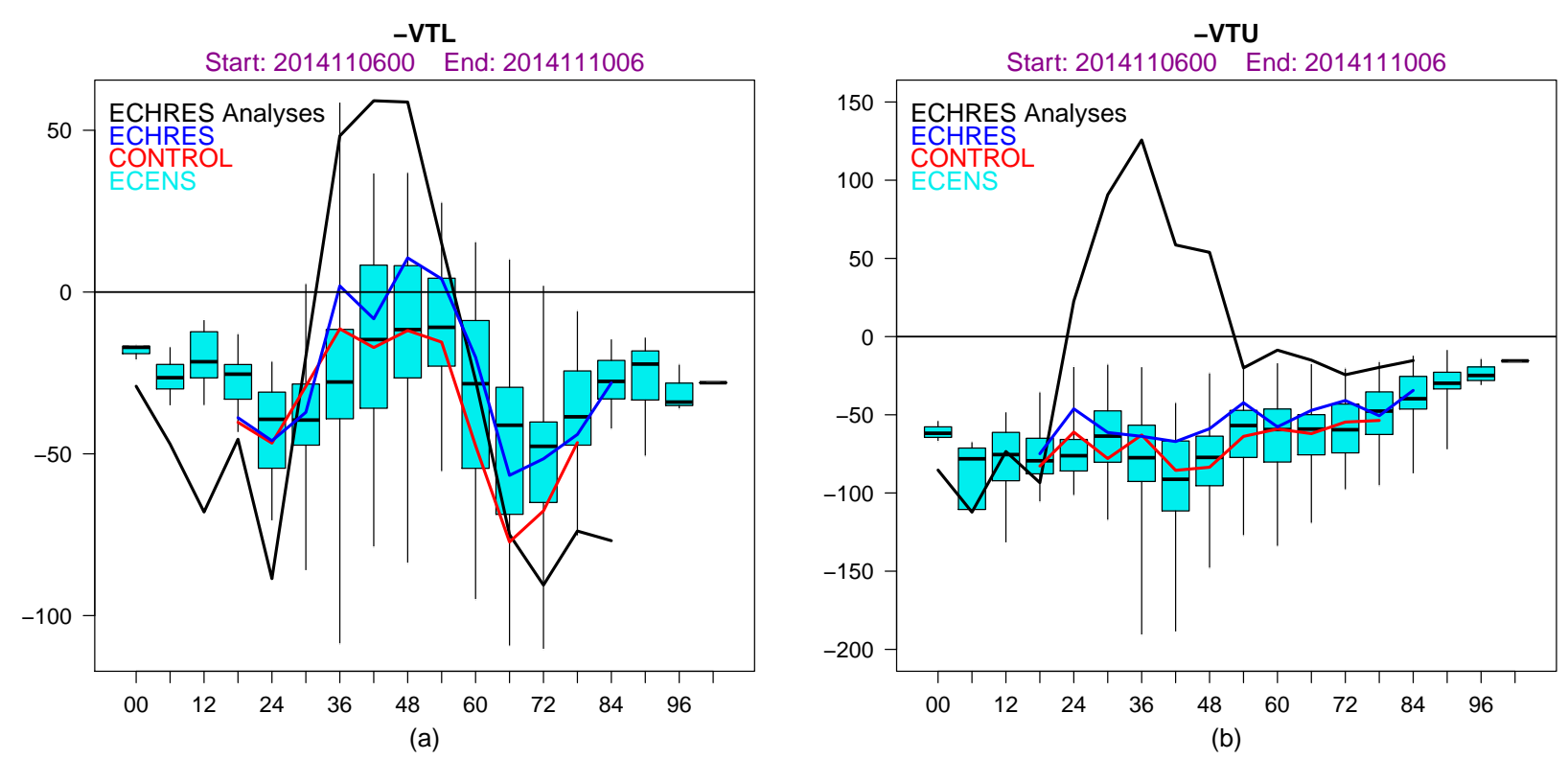

Figura 33.5: Como en la Figura 33.4 en la página anterior, ahora para el $-V_{T}^{L}$ (izquierda) y el -V $V_{U}^{L}($ derecha).

Los EPSgramas de la presión y de la intensidad muestran que los previstos son capaces de simular el periodo de máxima profundidad del ciclón, si bien la intensidad es mucho menor que la analizada. Algunos miembros del ensemble se aproximan más a los valores observados, incluso algún valor extremo (atípico, en inglés outlier) asigna un gradiente de presión mayor al analizado.

En este caso, la Figura 33.4 en la página anterior está informando de que se espera un ciclón que probablemente será muy intenso, con una caída de presión en su centro que puede ser de hasta $10 \mathrm{hPa}$ en 36 horas y no descarta la posibilidad (con una probabilidad muy baja) de que sea aún mayor. Los valores previstos por el ECHRES son muy similares a los previstos por el miembro de control y aunque coinciden con el análisis en los momentos de máxima intensidad, no son capaces de predecir la severidad de la evolución del ciclón. No predicen correctamente la caída de presión que muestra el análisis, sino que prevén una variación de presión central mucho más suave, con más de $11 \mathrm{hPa}$ de diferencia en el momento de menor presión.

En cuanto a la evolución de la estructura térmica del ciclón, según los EPSgramas de la Figura 33.5 y los DF en la Figura 33.6 en la página siguiente se prevé un ciclón simétrico pero de núcleo frío, excepto en un corto periodo de tiempo. Insinúa ligeramente el desarrollo del núcleo cálido de nivel bajo, pero sin conseguirlo. Algunos miembros son capaces de predecir un núcleo cálido a nivel bajo, pero en ningún caso con suficiente profundidad. Para estos parámetros sí se observa cierta diferencia entre el ECHRES y el miembro de control. El ECHRES simula un ciclón simétrico pero de núcleo frío, excepto en un corto periodo de tiempo en el que aparece un núcleo cálido somero, con valores muy inferiores a los analizados, que en ningún momento aparece en la evolución simulada por el control.

A la vista de estos resultados, podemos concluir que los modelos ECHRES y ECENS detectan la presencia del ciclón, principalmente en los momentos centrales de su ciclo de vida y son capaces de reproducir su trayectoria, aunque con algunos errores. Estos modelos identifican los momentos en los que el ciclón es más intenso, pero ni el ECHRES ni el miembro de control simulan la abrupta caída de presión en el centro del ciclón, ni los fuertes gradientes de presión que la acompañan. Sólo un pequeño porcentaje de miembros del ensemble avisa de la posibilidad de desarrollo de un ciclón de intensidad extraordinaria. En cuanto a la predicción de la estructura térmica del medicane, solamente algunos miembros del ensemble (no el control) y en menor medida el ECHRES son capaces de predecir un núcleo cálido en la capa baja, poco marcado y de corta duración, y en ningún caso en la capa alta. Sin duda este es uno de los aspectos a mejorar. Todo ello muestra que, a pesar de las limitaciones que tienen ambos modelos, solamente la predicción probabilista alerta, aunque asignándole baja probabilidad, de la posible formación de un ciclón extraordinariamente intenso con algunas características propias de un medicane. 

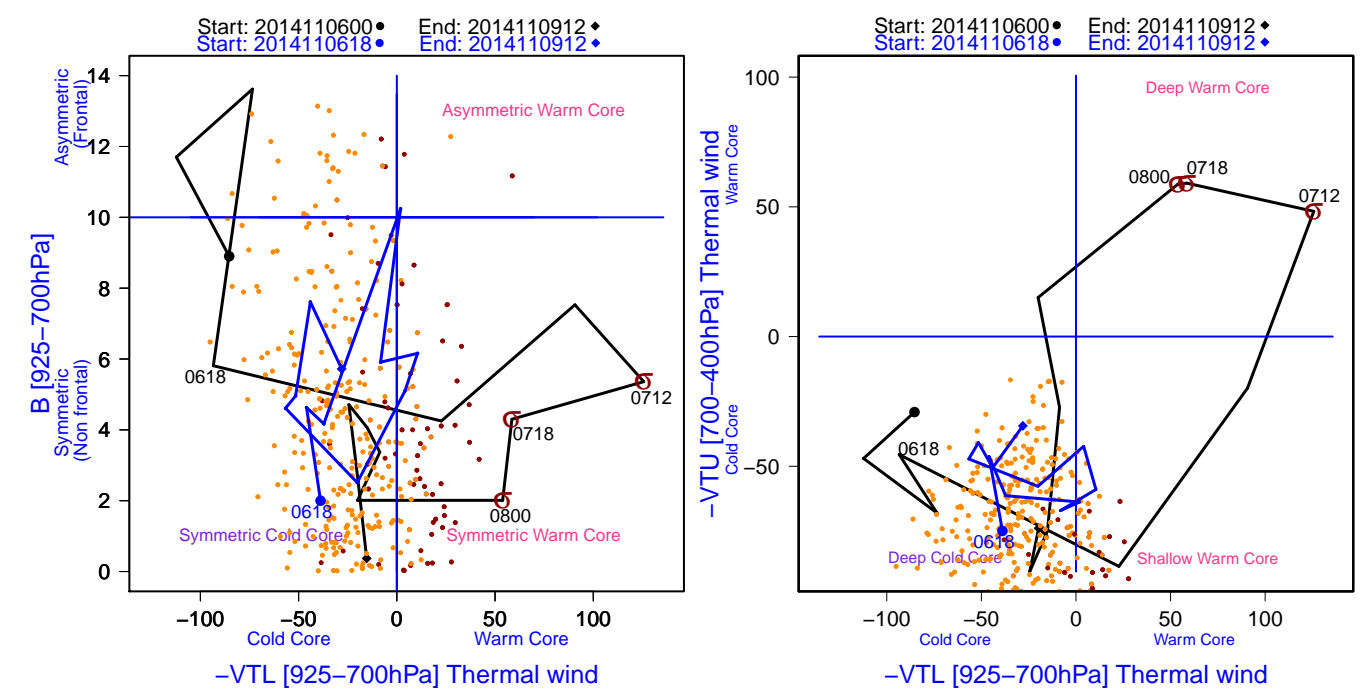

Figura 33.6: Diagramas de fase I y II. Se muestra la evolución analizada (en negro) y prevista (en azul) por el ECHRES. Los puntos corresponden a los ciclones previstos por el ECENS en el momento que son intensos (en naranja) o muy intensos (en rojo). El símbolo $\sigma$ indica que el ciclón se identifica como medicane.

\subsection{Conclusiones}

En este capítulo hemos mostrado diferentes procedimientos para detectar, seguir y caracterizar de una manera objetiva los ciclones mediterráneos a partir de modelos numéricos. Estas herramientas se pueden aplicar tanto a los campos analizados como previstos. El análisis puede suponer una buena aproximación a la "realidad" y puede utilizarse tanto para el diagnóstico como para la verificación de las predicciones, tanto deterministas como probabilistas.

La predicción de los ciclones mediterráneos relacionados con tiempo adverso puede ser muy útil para los encargados de realizar las predicciones y de emitir avisos. La ventaja de utilizar las predicciones probabilistas sobre las deterministas está suficientemente explicada en este libro y supone un reto a la hora de mostrar de forma clara y concisa la enorme cantidad de información que contiene.

Hoy en día existen muchos productos destinados a la predicción de los ciclones, sobre todo los tropicales. Aquí se presentan algunos de los posibles productos que se pueden utilizar para la predicción de los ciclones mediterráneos en general, aunque nos hemos centrado en un tipo particular de ciclones como son los medicanes. Para ello hemos utilizado el ensemble global del ECENS y lo hemos hecho para un caso de 2014. El ECENS ha mejorado desde entonces, por ejemplo ha aumentado su resolución horizontal de 32 a $18 \mathrm{~km}$, por lo que seguramente también ha mejorado la predicción de este tipo de ciclones pequeños e intensos.

A la vista de este único caso, podemos aventurar que los modelos que hemos utilizado hasta el momento son capaces de predecir de manera aceptable (aunque mejorable, dada la diferencia que hay con el análisis; en cuanto a estructura térmica, no se ha realizado un estudio exhaustivo con ECENS) la intensidad y la trayectoria de ciclones muy intensos de pequeñas dimensiones, aunque tendremos que esperar a disponer de modelos aún mejores para poder predecirlos de manera adecuada, en especial su estructura térmica y sus posibles transiciones tropicales o extratropicales. Por ejemplo, la predicción retrospectica realizada con el modelo WFR y tomada como control en Carrió et al., 2017 [3], da una predicción de intensidad más exacta que ECENS para el caso presentado. La resolución (32 km ECENS, 2 km WRF) juega un papel fundamental.

Aplicar estas herramientas utilizando el nuevo sistema(s) de predicción por conjuntos (SPC) que se está desarrollando en AEMET, el AEMET- $\gamma$ SREPS (cap. 22 en la página 333), puede resultar idóneo para simular posibles ciclones mediterráneos y, especialmente, para la predicción de medicanes, dado que jugarán a favor tanto el factor resolución horizontal como el factor enfoque probabilista. 


\subsection{Referencias}

[1] Campins, Joan, Jansà, Agustí y GenoVÉS, Ana. "Three-dimensional structure of western Mediterranean cyclones". En: International Journal of climatology 26.3 (2006), páginas 323-343. DOI: 10.1002/joc. 1275 (citado en página 554).

[2] CAmPins, Joan y col. "Climatology of Mediterranean cyclones using the ERA-40 dataset". En: International Journal of Climatology 31.11 (2011), páginas 1596-1614. DOI: 10.1002/joc. 2183 (citado en página 552).

[3] CARRIÓ, D S y col. "Tropicalization process of the 7 November 2014 Mediterranean cyclone: Numerical sensitivity study". En: Atmospheric Research 197 (2017), páginas 300-312 (citado en páginas 558, 561).

[4] Cressman, George P. "An operational objective analysis system". En: Mon. Wea. Rev 87.10 (1959), páginas 367-374 (citado en página 554).

[5] Emanuel, K. "Genesis and maintenance of Mediterranean hurricanes". En: Advances in Geosciences 2.2 (2005), páginas 217-220. DOI: 10 . 5194/adgeo-2-217-2005 (citado en página 552).

[6] FRoude, L.S.R. "Prediction of extratropical cyclones by the TIGGE ensemble prediction systems". En: ECMWF Newsletter 125.Autumn 2012 (2010), páginas 22-29 (citado en página 553).

[7] Genovés, Ana y Jansà, Agustí. "Diabatic processes contribution to the November 2001 Storm". En: 4th EGS Plinius Conference on Mediterranean Storms. Mallorca: Universitat de les Illes Balears, 2003 (citado en página 552).

[8] Haiden, T y col. Evaluation of ECMWF forecasts, including the 2016 resolution upgrade. European Centre for Medium Range Weather Forecasts, 2016 (citado en página 553).
[9] HART, Robert E. "A cyclone phase space derived from thermal wind and thermal asymmetry". En: Monthly weather review 131.4 (2003), páginas 585-616 (citado en páginas 554, 555).

[10] JANSÀ, Agustin y col. "Western Mediterranean cyclones and heavy rain. Part 2: Statistical approach". En: Meteorological Applications 8.1 (2001), páginas 43-56 (citado en página 553).

[11] JANSÀ, Agustí y col. "MEDEX: a general overview". En: Natural Hazards and Earth System Sciences 14.8 (2014), páginas 1965-1984 (citado en página 552).

[12] Lionello, Piero y col. "Cyclones in the Mediterranean region: climatology and effects on the environment". En: Developments in Earth and Environmental Sciences 4 (2006), páginas 325-372 (citado en página 552).

[13] NeU, Urs y col. "IMILAST: A community effort to intercompare extratropical cyclone detection and tracking algorithms". En: Bulletin of the American Meteorological Society 94.4 (2013), páginas 529-547 (citado en página 554).

[14] Persson, Anders. "User guide to ECMWF forecast products". En: Ecmwf March (2011), página 127 (citado en página 556).

[15] Picornell, M A, CAmpins, Joan y JanSÀ, Agustí. "Detection and thermal description of medicanes from numerical simulation". En: Natural Hazards and Earth System Sciences 14.5 (2014), página 1059 (citado en página 555$)$.

[16] Picornell, M A, Jans À, Agustí y GenoVÉS, Ana. "A tool for assessing the quality of the Mediterranean cyclone forecast: a numerical index". En: Natural Hazards and Earth System Sciences 11.6 (2011), páginas 1787-1794 (citado en página 554).

[17] Picornell, M A y col. "Automated database of mesocyclones from the HIRLAM (INM)- $0.5^{\circ}$ analyses in the western Medite- 
rranean". En: International journal of climatology 21.3 (2001), páginas 335-354. DOI: 10.1002/joc. 621 (citado en página 554).

[18] Ramis, C. y col. Medicanes: ciclones cuasitropicales mesoescalares en el Mediterráneo. Editado por GARCíA-LEGAZ, Carlos y VALERO, Francisco. 2013 (citado en página 552).

[19] UPPAlA, Sakari M y col. "The ERA-40 reanalysis". En: Quarterly Journal of the royal meteorological society 131.612 (2005), páginas 2961-3012. DOI: 10.1256/qj . 04.176 (citado en página 554). 
